\title{
The Impact of Penicillin Skin Testing on Clinical Practice and Antimicrobial Stewardship
}

\author{
Ramzy H. Rimawi, MD, Paul P. Cook, MD¹, Michael Gooch, MS, RPh², Badih Kabchi, MD¹, Muhammad S. Ashraf, MD, \\ Bassam H. Rimawi, MD³ ${ }^{3}$, Mulugeta Gebregziabher, $\mathrm{PhD}^{4}$, Dawd S. Siraj, MD, MPH\&TM ${ }^{1}$
}

${ }^{1}$ Department of Internal Medicine, Division of Infectious Diseases, Brody School of Medicine-East Carolina University, Greenville, NC; ${ }^{2}$ Department of Pharmacy, Vidant Medical Center, Greenville, NC; ${ }^{3}$ Division of Reproductive Infectious Diseases, Medical University of South Carolina, Charleston, SC; ${ }^{4}$ Department of Biostatistics, Medical University of South Carolina, Charleston, SC.

BACKGROUND: Penicillin skin testing (PST) is a simple and reliable way of diagnosing penicillin allergy. After being off the market for 4 years, penicilloyl-polylysine was reintroduced in 2009 as PRE-PEN. We describe the negative predictive value (NPV) of PST and the impact on antibiotic selection in a sample of hospitalized patients with a reported history of penicillin allergy.

METHODS: We introduced a quality improvement process at our 861-bed tertiary care hospital that used PST to guide antibiotic usage in patients with a history consistent with an immunoglobulin E (lgE)-mediated reaction to penicillin. Subjects with a negative PST were then transitioned to a $\beta$-lactam agent for the remainder of their therapy. NPV of skin testing was established at 24-hour follow-up. We are reporting the result of 146 patients tested between March 2012 and July 2012.
RESULTS: A total of 146 patients with a history of penicillin allergy and negative PST were treated with $\beta$-lactam antibiotics. Of these, only 1 subject experienced an allergic reaction to the PST. The remaining 145 patients tolerated a full course of $\beta$-lactam therapy without an allergic response, giving the PST a 100\% NPV. We estimated that PST-guided antibiotic alteration for these patients resulted in an estimated annual savings of $\$ 82,000$.

CONCLUSION: Patients with a history of penicillin allergy who have a negative PST result are at a low risk of developing an immediate-type hypersensitivity reaction to $\beta$-lactam antibiotics. The increased use of PST may help improve antibiotic stewardship in the hospital setting. Journal of Hospital Medicine 2013;8:341-345. (c) 2013 Society of Hospital Medicine
Self-reported penicillin allergy is common and frequently limits the available antimicrobial agents to choose from. This often results in the use of more expensive, potentially more toxic, and possibly less efficacious agents. 1,2

For over 30 years, penicilloyl-polylysine (PPL) penicillin skin testing (PST) was widely used to diagnose penicillin allergy with a negative predictive value (NPV) of about $97 \%$ to $99 \%{ }^{3}$ After being off the market for 5 years, PPL PST was reapproved in 2009 as PRE-PEN. ${ }^{4}$ However, many clinicians still fail to utilize PST despite its simplicity and substantial clinical impact. The main purpose of this study was to describe the predictive value of PST and impact on antibiotic selection in a sample of hospitalized patients with a reported history of penicillin allergy.

\section{METHODS}

In 2010, PST was introduced as a quality-improvement measure after approval and support from the

\footnotetext{
*Address for correspondence and reprint requests: Ramzy H. Rimawi, MD, Brody School of Medicine-East Carolina University, Doctor's Park 6A, Mail Stop 715, Greenville, NC 27834; Telephone: 252-744-4500; Fax: 252-744-3472; E-mail: ramzyrimawi@hotmail.com

Additional Supporting Information may be found in the online version of this article.

Received: December 27, 2012; Revised: February 27, 2013; Accepted: February 28, 2013

2013 Society of Hospital Medicine DOI 10.1002/jhm.2036

Published online in Wiley Online Library (Wileyonlinelibrary.com).
}

chief of professional services and the medical staff executive committee at Vidant Medical Center, an 861bed tertiary care and teaching hospital. Our antimicrobial stewardship program is regularly contacted for approval of alternative therapies in penicillin allergic patients. The PST quality-improvement intervention was implemented to avoid resorting to less appropriate therapies in these situations. Following approval by the University and Medical Center Institutional Review Board, we designed a 4-month study to assess the impact of this ongoing quality improvement measure from March 2012 to July 2012.

Hospitalized patients of all ages with reported penicillin allergies were obtained from our antimicrobial stewardship database. Their charts were reviewed for demographics, antibiotic use, clinical infection, and allergic description. Deciding whether to alter antibiotic therapy to a $\beta$-lactam regimen was based on microbiologic results, laboratory values, clinical infection, and history of immunoglobulin $\mathrm{E}(\mathrm{IgE})$-mediated reactions, as defined by the updated drug allergy practice parameters. ${ }^{5}$ IgE-mediated reactions included: (1) immediate urticaria, laryngeal edema, or hypotension; (2) anemia; and (3) fever, arthralgias, lymphadenopathy, and an urticarial rash after 7 to 21 days. $^{5-7}$ We defined anaphylaxis as the development of angioedema or hemodynamic instability within 1 hour of penicillin administration. A true negative reaction was a lack of an IgE-mediated reaction to all the drug challenges. 


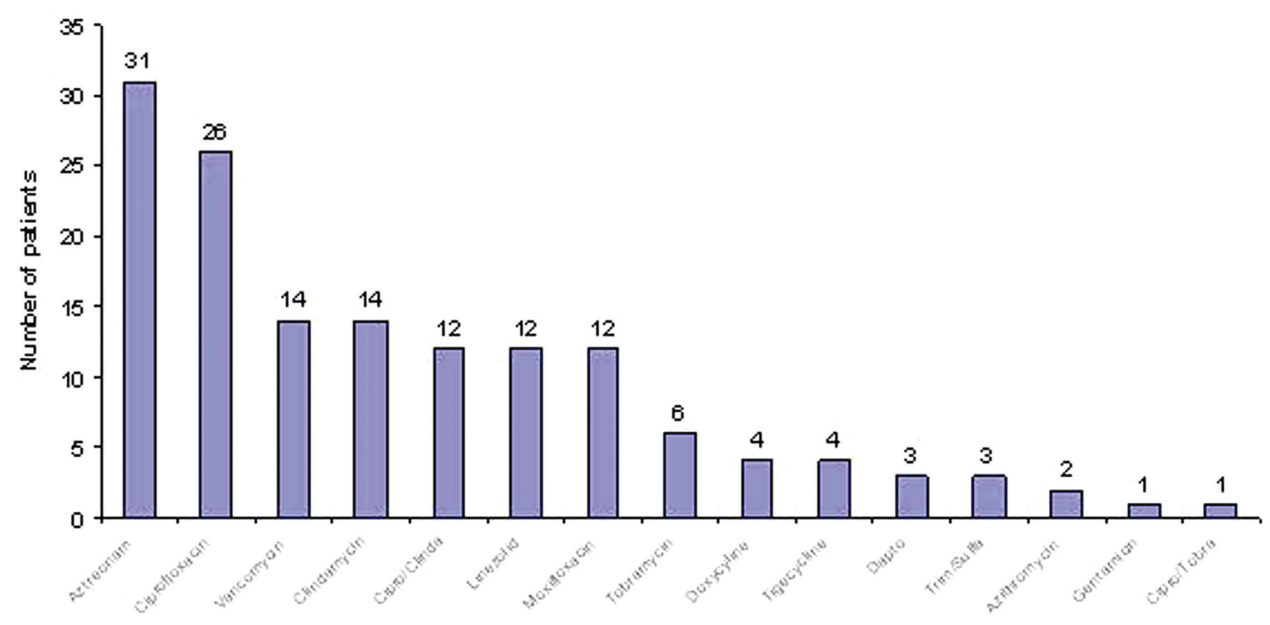

Antibiotics Given After Hegative Penicillin Skin Test

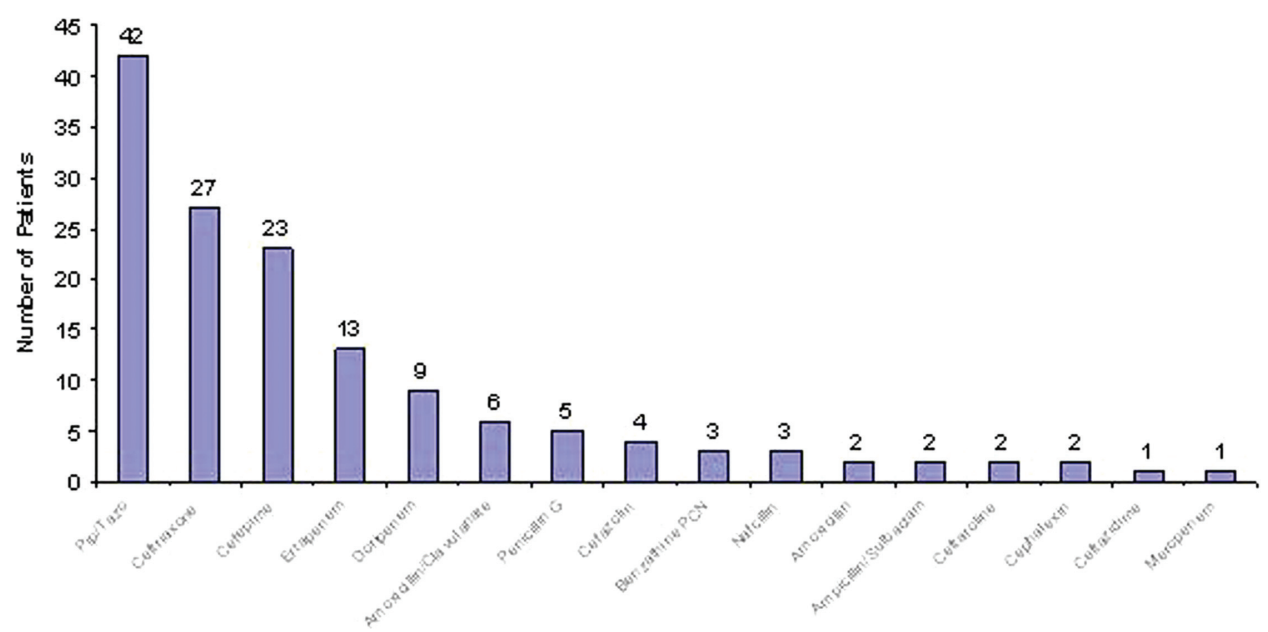

FIG. 1. Antibiotics used prior to penicillin skin testing and $\beta$-lactams transitioned to after a negative penicillin skin test. The upper graph illustrates the antibiotics used prior to penicillin skin testing in 146 patients over the 5-month study period. The lower graph illustrates the $\beta$-lactam antibiotics used after a negative penicillin skin test in the same patients. Abbreviations: Cipro, ciprofloxacin; Clinda, clindamycin; Dapto, daptomycin; Pip/Tazo, piperacillin-tazobactam; Tobra, tobramycin; Trim/Sulfa, trimethoprim-sulfamethoxazole.

Patients in the medical, surgical, labor, and delivery wards; intensive care units; and emergency department underwent testing. The $\beta$-lactam agent used after a negative PST was recorded, and the patients were followed for 24 hours after transitioning their therapy to a $\beta$-lactam regimen. Excluded subjects included those with (1) non-IgE-mediated reactions, (2) skin conditions that can give false positive results, (3) medications that may interfere with anaphylactic therapy, (4) history of severe exfoliative reactions to $\beta$-lactams, (5) anaphylaxis less than 4 weeks prior, (6) allergies to antibiotics other than penicillin, and (7) uncertain allergy history.

\section{PST Reagents/Procedure}

Our benzylpenicilloyl major determinant molecule, commercially produced as PPL, was purchased as a PRE-PEN from ALK-Abello, Round Rock, Texas.
Penicillin G potassium, purchased from Pfizer, New York, New York, is the only commercially available minor determinant and can improve identification of

TABLE 1. Prevalence of Reported Antimicrobial Drug Allergy in 4031 Charts Reviewed Over a 5-Month Period

\begin{tabular}{lcc}
\hline Antibiotic & $\begin{array}{c}\text { No. of Patients } \\
\text { Reporting An Allergy }\end{array}$ & $\begin{array}{c}\text { \% Per Total } \\
\text { Charts Reviewed }\end{array}$ \\
\hline Penicillin & 428 & 10.6 \\
Sulfonamide & 271 & 6.7 \\
Quinolone & 108 & 2.7 \\
Cephalosporin & 81 & 2.0 \\
Macrolide & 65 & 1.6 \\
Vancomycin & 39 & 0.9 \\
Tetracycline & 20 & 0.5 \\
Clindamycin & 18 & 0.4 \\
Metronidazole & 9 & 0.2 \\
Linezolid & 2 & 0.05 \\
\hline
\end{tabular}




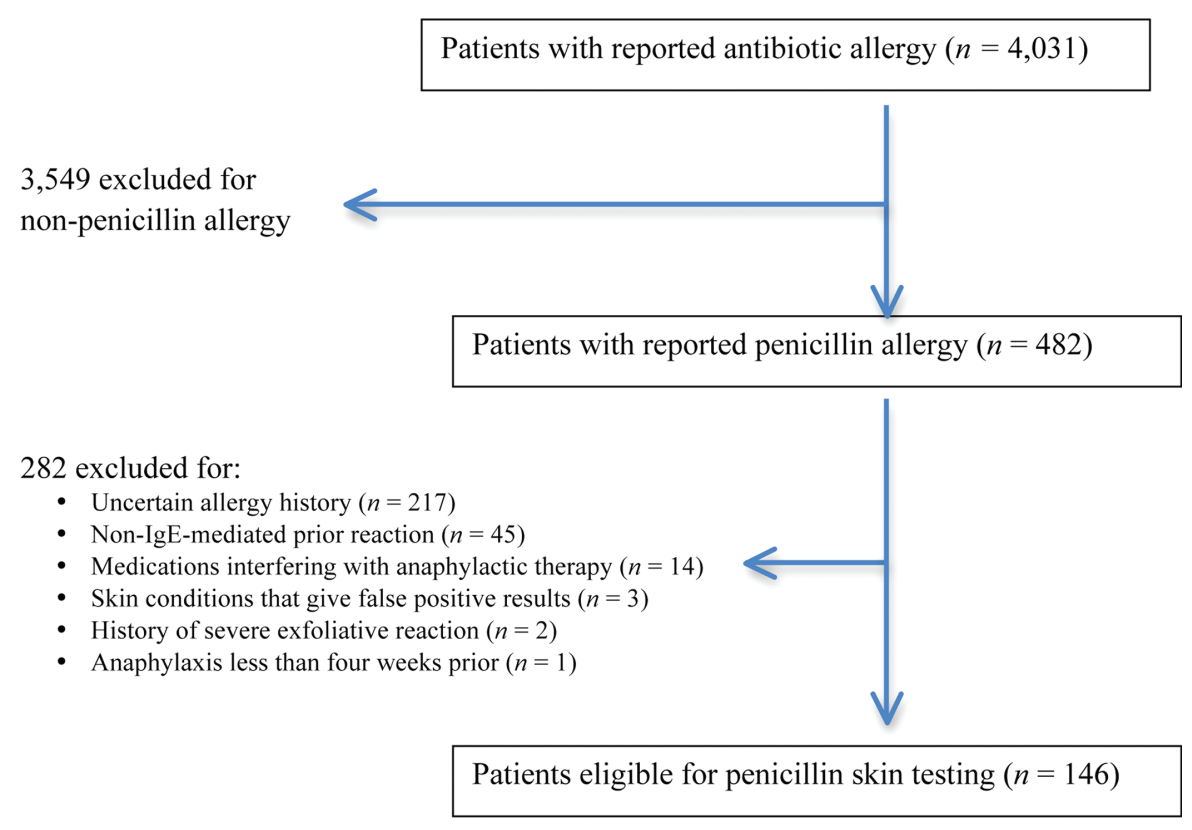

FIG. 2. Study design with inclusion and exclusion criteria. Abbreviations: IgE, immunoglobulin E.

penicillin allergy by up to $97 \% .^{2}$ The PST panel also included histamine (positive control) and normal saline (negative control).

\section{Skin Testing Procedure}

An infectious diseases fellow (R.H.R. or B.K.) was supervised in preparing for potential anaphylaxis, applying the reagents and interpreting the results based on drug allergy practice parameters. ${ }^{5}$ The preliminary epicutaneous prick/puncture test was performed with a lancet in subjects without prior anaphylaxis using full-strength PPL and penicillin G potassium reagents. If there was no response within 15 minutes, which we defined as a lack of wheal formation $\geq 3 \mathrm{~mm}$ or greater than that of the negative control, 0.02 to $0.03 \mathrm{~mL}$ of each reagent was injected intradermally using a tuberculin syringe and examined for 15 minutes. ${ }^{5}$ If there was no response, patients were then challenged with either a single oral dose of penicillin $\mathrm{V}$ potassium $250 \mathrm{mg}$ or whichever oral penicillin agent they previously reported an allergy to. If no reaction was appreciated within 2 hours, their therapy was changed to a $\beta$-lactam agent including penicillins, cephalosporins, and carbapenems for the remaining duration of therapy (Figure 1) An estimate of NPV was obtained after 24 hours follow-up.

\section{Statistical Analysis}

We designed a study to estimate whether the reapproved PST achieves an NPV of at least $95 \%{ }^{3}$ We hypothesized that clinicians will be willing to utilize PST even if it has an NPV of slightly less than $98 \%$ compared to the current standard of treating patients without PST. 7 Assuming an equivalence margin of $3 \%$, we estimated a sample size of 146 to achieve at least $82 \%$ power to test a hypothesis of NPV $\leq 95 \%$ using a 1-sided $Z$ test with a type-I error rate of $5 \% .{ }^{8}$ Once the sample size of 146 subjects was reached, we stopped recruiting patients.

Sample characteristics of the subjects who underwent testing were summarized using descriptive statistics. Sample proportions were calculated to summarize categorical variables. Mean and standard deviation were calculated to summarize continuous variables. Cost analysis of antibiotic therapy was estimated from the Vidant Medical Center antibiotic pharmaceutical acquisition costs. Estimated cost of peripherally inserted central catheter (PICC) placement and removal as well as laboratory testing costs were obtained from our institution's medical billing department. Marketing costs of pharmacist drug calibration and nursing assessments with dressing changes were obtained from hospital-affiliated outpatient antibiotic infusion companies.

\section{RESULTS}

A total of 4031 allergy histories were reviewed during the 5-month study period to achieve the sample size of 146 patients (Table 1). Of those, 3885 were excluded (Figure 2). Common infections included pneumonias $(26 \%)$ and urinary tract infections $(20 \%)$ (Table 2) Only 1 subject had a positive reaction with hives, edema, and itching approximately 6 minutes after the agents were injected intradermally. The remaining $145(99 \%)$ had negative reactions to the PST and oral challenge and were then successfully transitioned to a $\beta$-lactam agent without any reaction at 24 hours, giving an NPV of $100 \%$. Ten subjects were switched from intravenous to oral $\beta$-lactam agents (Figure 1). Avoidance of PICC placement 


\begin{tabular}{|c|c|}
\hline Categories & No. of Patients (\%) \\
\hline \multicolumn{2}{|l|}{ Time since last reported penicillin use } \\
\hline 1 month-1 year & $6(4)$ \\
\hline $2-5$ years & $39(27)$ \\
\hline $6-10$ years & $23(16)$ \\
\hline$>10$ years & $78(53)$ \\
\hline \multicolumn{2}{|l|}{ Reported lgE-mediated reactions } \\
\hline Bronchospasm & $23(16)$ \\
\hline Urticarial rash & $100(68)$ \\
\hline Edema & $32(22)$ \\
\hline Anaphylaxis & 21 (14) \\
\hline \multicolumn{2}{|l|}{ Age on admission, y } \\
\hline $20-50$ & $28(19)$ \\
\hline $51-60$ & $29(20)$ \\
\hline $61-70$ & $41(28)$ \\
\hline $71-80$ & $24(16)$ \\
\hline$>80$ & $24(16)$ \\
\hline \multicolumn{2}{|l|}{ Gender } \\
\hline Male & $55(40)$ \\
\hline Female & $88(60)$ \\
\hline \multicolumn{2}{|l|}{ Race } \\
\hline White & $82(56)$ \\
\hline Black & $61(42)$ \\
\hline Hispanic & $3(2)$ \\
\hline \multicolumn{2}{|l|}{ Infections being treated } \\
\hline Bacteremia & $7(4.8)$ \\
\hline Catheter-related bloodstream infection & $2(1.4)$ \\
\hline Empyema & $1(0.7)$ \\
\hline Epidural abscess & $2(1.4)$ \\
\hline Infective endocarditis & $4(2.7)$ \\
\hline Intra-abdominal infection & $24(16.4)$ \\
\hline Meningitis & $1(0.7)$ \\
\hline Neutropenic fever & $1(0.7)$ \\
\hline Osteomyelitis & $6(4.1)$ \\
\hline Pericardial effusion & $1(0.7)$ \\
\hline Prosthetic joint infection & $5(3.4)$ \\
\hline Pneumonia & $40(27.4)$ \\
\hline Skin and soft-tissue infection & $20(13.7)$ \\
\hline Syphilis & $3(2.1)$ \\
\hline Urinary tract infection & $29(19.7)$ \\
\hline
\end{tabular}

NOTE: Abbreviations: IgE, Immunoglobulin E.

$(\$ 1,200)$ and removal $(\$ 65)$, dressing changes, weekly drug-level testing, laboratory technician, and pharmaceutical drug calibration costs allowed for a healthcare reduction of $\$ 5,233$ ( $\$ 520 /$ patient) based on the 146 patients studied. The total cost of therapy would have been $\$ 113,991$ if the PST had not been performed. However, the cost of altered therapy following a negative PST was $\$ 81,180$, a difference of $\$ 32,811$ (\$225/per patient) in a 5-month period. The total estimated annual difference, including antibiotic alteration and associated drug-costs, would be $\$ 82,000$.

\section{DISCUSSION}

PST is the most rapid, sensitive, and cost-effective modality for evaluating patients with immediate allergic reactions to penicillin. Over $90 \%$ of individuals with a true history of penicillin allergy have confirmed sensitivity with a PST, implying most patients who are skin tested negative are truly not allergic. ${ }^{7,9-12}$ Our study shows that the reapproved PST with the PPL and penicillin $G$ determinants continues to have a high NPV. A patient with a negative PST result is generally at a low risk of developing an immediate-type hypersensitivity reaction to penicillin. ${ }^{2,11}$ PST frequently allowed for less expensive agents that would have been avoided due to a reported allergy. The estimated annual savings of $\$ 82,000$ dollars from antibiotic alteration with successful transition to a $\beta$-lactam agent after a negative PST illustrates its value, supports its validity, and makes this study novel.

Many $\beta$-lactamase inhibitors (ie, piperacillin-tazobactam), fourth generation cephalosporins (ie, cefepime), and carbapenems still remain costly. Despite this, we were still able to achieve a significant reduction in overall cost. In addition to financial benefits, PST allowed for the use of more appropriate agents with less potential adverse effects. Narrow-spectrum, non- $\beta$-lactam agents were sometimes altered to a broader-spectrum $\beta$-lactam agent. We also frequently tailored 2 agents to just 1 broad-spectrum $\beta$-lactam. This led to more patients being given broad-spectrum agents after the PST (72 vs 89 patients). However, we were able to avoid using second-line agents, such as aztreonam, vancomycin, linezolid, daptomycin, and tobramycin, in many patients with infections that are often best treated with penicillin-based antibiotics (ie, syphilis, group B Streptococcus infections). With increasing incidence and recovery of multidrug-resistant bacteria, PST may also allow use of potentially more effective antimicrobial agents.

A possible limitation is that our prevalence of a true penicillin allergy was $<1 \%$, whereas Bousquet et al. illustrate a higher prevalence of about $20 \%{ }^{7}$ Although our prevalence may not be generalizable, Bousquet's study only assessed patients with allergies $<5$ years prior.

The introduction of PST into clinical practice will allow trained healthcare providers to prescribe cheaper, more appropriate, less toxic antimicrobial agents. The overall benefit of reintroducing penicillin agents when needed in the future is far more costeffective than what is described here. PST should become a standard of care when prescribing antibiotics to patients with a history of penicillin allergy. Medical providers should be aware of its utility, acquire training, and incorporate it into their practice.

Disclosures: Paul P. Cook, MD, has potential conflicts of interest with Gilead (investigator), Pfizer (investigator), Merck (investigator and speakers' bureau), and Forest (speakers' bureau). Neither he nor any of the other authors has received any sources of funding for this article. For the remaining authors, no conflicts were declared. The corresponding author, Ramzy Rimawi, MD, had full access to all of the data in the study and had final responsibility for the decision to submit for publication.

\section{References}

1. Jost BC, Wedner HJ, Bloomberg GR. Elective penicillin skin testing in a pediatric outpatient setting. Ann Allergy Asthma Immunol. 2006;97(6):807-812. 
2. US Department of Veterans Affairs Web site. Benzypenicilloyl polylisine (PRE-PEN) national drug monograph. May 2012. Available at: http://www.pbm.va.gov/DrugMonograph.aspx. Accessed September 1, 2012.

3. Park M, James T. Diagnosis and management of penicillin allergy. Mayo Clin Proc. 2005;80(3):405-410.

4. PRE-PEN penicillin skin test antigen. Available at: http://www.alkabello.com/us/products/pre-pen/Pages/PREPEN.aspx. Accessed September 1, 2012.

5. Solensky R, Khan DA, Bernstein IL, et al.; Joint Task Force on Practice Parameters; American Academy of Allergy, Asthma and Immunology; American College of Allergy, Asthma and Immunology; Joint Council of Allergy, Asthma and Immunology. Drug allergy: an updated practice parameter. Ann Allergy Asthma Immunol. 2010;105:259-273.

6. Parker CW. Immunochemical mechanisms in penicillin allergy. Fed Proc. $1965 ; 24: 51-54$.
7. Bousquet PJ, Pipet A, Bousquet-Rouanet L, et al. Oral challenges are needed in the diagnosis of beta-lactam hypersensitivity. Clin Exp Allergy. 2008;38(1):185-190.

8. Chow SC, Shao J, Wang H. Sample Size Calculations in Clinical Research. New York, NY: Chapman \& Hall/CRC; 2003.

9. Richter AG, Wong G, Goddard S, et al. Retrospective case series analysis of penicillin allergy testing in a UK specialist regional allergy clinic. J Clin Pathol. 2011;64:1014-1018.

10. Stember RH. Prevalence of skin test reactivity in patients with convincing, vague and unnacceptible histories of penicillin allergy. Allergy Asthma Proc. 2005;26(1):59-64.

11. Valyasevi MA, Van Dellen RG. Frequency of systemic reactions to penicillin skin tests. Ann Allergy Asthma Immunol. 2000;85: 363-365.

12. Lee CE, Zembower TR, Fotis MA, et al. The incidence of antimicrobial allergies in hospitalized patients. Arch Intern Med. 2000;160;2819-2822. 